\title{
The effect of the orienting reaction on disjunctive reaction time'
}

Eighty Ss performed a visual or auditory disjunctive reaction time (DRT) task in which some of the task stimuli were preceded, at irregular intervals, by an intense visual or auditory "orienting stimulus." Initially the orienting stimuli impaired speed of response, but on subsequent presentations produced shorter DRTs than when no orienting stimulus was given. It is suggested that the warning signal used in RT experiments may influence performance not only as a result of the information it carries, but also because it may elicit an orienting reaction.

In a simple RT experiment shorter RTs are generally found when a warning signal is used than when no warning is given, provided that the interval between the warning and task stimuli is constant. If the warning interval is irregularly varied, no difference is found (Hermelin \& Venables, 1964; Klemmer, 1956). An apparent exception to these results is found in a study by John (1964). He showed that the speed of response to auditory task stimuli was facilitated when they were preceded, at irregular intervals, by an extraneous visual stimulus. It is possible that the conflicting findings are partly due to the confounding of two properties of the warning signal. Although intended to inform the $\mathrm{S}$ of the impending task stimulus, the warning signal may also act as an "orienting stimulus," giving rise to the orienting reaction, which has been shown to affect certain sensory and perceptual processes (e.g., Martin \& Edelberg, 1963; Sokolov, 1963).

A test of the hypothesis that the orienting reaction, which results from the presentation of the warning signal, improves performance in an RT experiment would require that the informational properties of the warning signal be eliminated, so that it would serve merely as an "orienting stimulus." In the present experiment the temporal relation between the warning (now orienting) stimulus and the task stimulus were arranged to be so unpredictable as to minimize the informational value of the former. The effects of such non-informational auditory and visual stimuli on the speed of disjunctive response to auditory and visual task stimuli were studied.

\section{Method}

The Ss were 80 male volunteers from the Armed Forces. Their ages ranged from 17 years to 47 years with a mean of 23.7 years.

$\mathrm{S}$ was seated facing into a dimly illuminated wooden cubicle. The visual task stimulus consisted of a $1 / 5 \mathrm{sec}$. flash of red or green light projected onto a screen situated at eye-level at a distance of 3 ft. from S. The auditory task stimulus was a $1 / 5 \mathrm{sec} .80 \mathrm{~dB} 500 \mathrm{cps}$ tone presented to S's right or left ear through earphones. The response unit consisted of two button-operated microswitches mounted 2 in. apart on the arm of S's chalr. A $1 / 5 \mathrm{sec}$. burst of $70 \mathrm{db}$ white nolse delivered to both ears served as the auditory orienting stimulus, and a $1 / 5 \mathrm{sec}$. burst of flashing white light was the visual orienting stimulus. The light, from a source situated directly above $\mathrm{S}$, had a frequency of 1000 flashes $/ \mathrm{min}$. and a peak intensity of 0.21 million candlepower.

Ss were randomly assigned to four equal groups, each group receiving one of the four possible combinations of auditory and visual orienting and task stimuli. The experiment consisted of three parts, run consecutively for all groups. In parts I and III, the two control series, each $S$ received 20 randomly ordered blank trials (no orienting stimulus). In part II each $S$ received four successive blocks of 10 trials, each block consisting of four critical trials on which the orienting stimulus preceded the task stimulus by $0,1,2$, or 4 sec., four blank trials, and two trials on which the interval between the orienting and task stimuli was 6 or $8 \mathrm{sec}$. The results for these last two trials, which were designed to make the orienting and task stimuli appear more independent, are not included in the analyses. The order of orienting stimulus-task stimulus intervals used on the four critical trials was balanced over blocks and Ss according to a Latin square design. The interval between successive task stimuli was 8,10 , or $12 \mathrm{sec}$, randomly ordered.

Ss were instructed to respond to the task stimuli by pressing the appropriate button as quickly as possible. Half the Ss in each of the two groups receiving visual task stimuli were told to press the right button for a red stimulus, the left button for a green stimulus; the remaining $S s$ had the reverse combination. Ss receiving the auditory task stimulus were instructed to respond to a tone in the left earphone by pressing the left button, and to a tone in the right earphone by pressing the right button. For half these Ss the earphones, but not the instructions, were reversed.

Results

The control series in parts I and III of the experiment were designed to detect effects of practice or fatigue. The average control DRTs indicated a general decrease, from part I to part III, in response speed for one group, and an increase for the other three groups. Two-tailed t-tests showed none of these changes to be significant.

For each group, the results of part II showed the average DRT for the four critical trials to be longer than that for the blank trials in Block 1, but the relation was reversed in later blocks. An analysis of variance showed this interaction between critical vs. blank DRT and blocks to be significant $(p<.001)$. These results, averaged over groups, appear in Fig. 1.

The average DRTs for the four orienting stimulustask stimulus intervals and for the blank trials are shown in Fig. 2. On the basis of the differential effect displayed in Fig. 1, the results shown are for Block 1 and the average of Blocks 2, 3, and 4 . The results for the 0 sec. interval for the group receiving auditory orienting and task stimuli have been omitted from the analysis. ${ }^{2}$

\section{Discussien}

Comparison of the average DRTs for the critical and blank trials shown in Fig. 1 indicates that the occurrence of the orienting stimulus impaired speed of response in Block 1. It seems likely that this initial (Block 1) impairment was due to interference caused by the occur- 


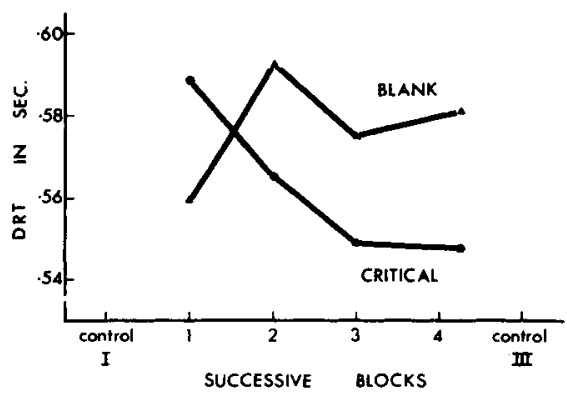

Fig. 1. Mean disjunctive reaction times for the critical and blank trials for each block in part $I I$, and for the control series in parts I and III.

rence of "novelty reactions," an effect previously demonstrated by Favreau (1964). It will be seen in Fig. 2 that the initial impairment was generally greater when the orienting stimulus was auditory, especially at the 1 and 2 sec. intervals. Although both orienting stimuli were intense, it is generally agreed (Sokolov, 1963) that auditory stimuli, per se, are likely to elicit stronger novelty or orienting reactions than are visual stimuli. The clearest evidence for facilitation was seen when both orienting and task stimuli were visual, this effect being most noticeable at the 1 and $2 \mathrm{sec}$. intervals.

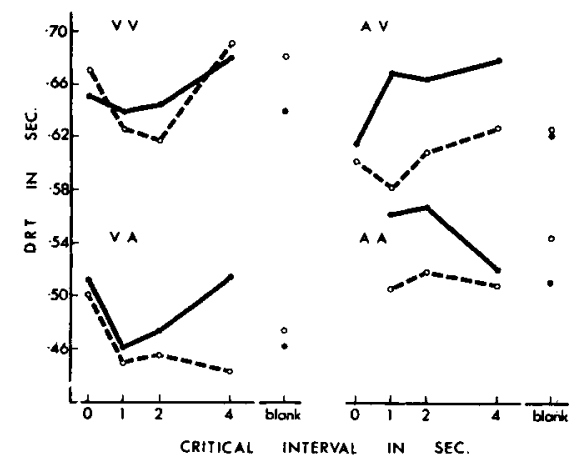

Fig. 2. Disjunctive reaction time as a function of interval between orienting and task stimuli for Block 1 (solid line) and the average of Blocks 2, 3 and 4 (broken line) for each group. The corresponding mean DRTs for the blank trials are also shown. A denotes auditory, $V$ denotes visual; the first letter of each pair refers to the orienting stimulus, the second letter refers to the task stimulus.
Although every attempt was made to prevent $S$ from associating the orienting and task stimuli, it is possible that the facilitation resulted from Ss regarding the orienting stimulus as a warning signal, thereby gaining some small amount of information as to the occurrence of the task stimulus. A more plausible interpretation, however, may be based on the progressive habituation of the orienting reaction. Thus the increase in arousal underlying the orienting reaction was initially sufficiently large to interfere with performance, but subsequently diminished until its effect was one of facilitation. Evidence which may be taken as support for this interpretation has been obtained by Fuster (1958) who stimulated the reticular formation of monkeys performing a visual discrimination task. He found that the shortest RTs were associated with medium levels of stimulation, higher levels resulting in poorer performance.

It is concluded that the effect of an orienting stimulus on DRT may be one of impairment or facilitation depending on the degree of habituation of the resulting orienting reaction. Inasmuch as a warning signal in an RT experiment may elicit an orienting reaction, its effect on performance may not be wholly determined by its informational value.

\section{References}

Favreau, 0. Proactive decremental effects on response speed in a continuous DRT task. Psychon. Sci., 1964, 1, 319-320.

Fuster, J. M. Effects of stimulation of brainstem on tachistoscopic perception. Science, 1958, 127, 150.

Hermelin, B. M. \& Venables, P. H. Reaction time and alpha blocking in normal and severely subnormal subjects. $J$. exp. Psychol., $1964,67,365-372$.

John, I. D. The role of extraneous stimuli in responsiveness to signals: refractoriness or facilitation? Aust. J. Psychol., 1964 , 16, 87-96.

Klemmer, E. I. Time uncertainty in simple reaction time. J. exp. Psychol., 1956, 51, 179-184.

Martin, R. D., \& Edelberg, R. The relationship of skin resistance changes to receptivity. J. Psychosom. Res., 1963, 7, 173-179.

Sokolov, Ye. N. Perception and the conditioned reflex. New York: Pergammon Press, 1963.

\section{Notes}

1. This research was supported by a grant (9425-10) from the Defence Research Board of Canada to Dr. Dalbir Bindra.

2. The average DRT on these trials was almost twice that for the other critical intervals. It was found that in this condition Ss had difficulty determining in which ear the task stimulus occurred. For this reason the results were not included in the analysis. 